\title{
Switching Characteristics of Ferroelectric Transistor Inverters
}

\section{Crystal Laws ${ }^{1}$, Cody Mitchell ${ }^{1}$, Todd C. MacLeod ${ }^{2}$, and Fat D. Ho ${ }^{1}$}

${ }^{1}$ The University of Alabama in Huntsville, Department of Electrical and Computer Engineering, Huntsville, Alabama, 35899, USA

${ }^{2}$ National Aeronautics and Space Administration, National Space Science and Technology Center, Huntsville, Alabama, 35805, USA

\begin{abstract}
This paper presents the switching characteristics of an inverter circuit using a ferroelectric field effect transistor, FeFET. The propagation delay time characteristics, $\tau_{\mathrm{phl}}$ and $\tau_{\mathrm{plh}}$ are presented along with the output voltage rise and fall times, $\tau_{\text {rise }}$ and $\tau_{\text {fall }}$. The propagation delay is the timedelay between the $\mathrm{V}_{50 \%}$ transitions of the input and output voltages. The rise and fall times are the times required for the output voltages to transition between the voltage levels $\mathrm{V}_{10 \%}$ and $\mathrm{V}_{90 \%}$. Comparisons are made between the MOSFET inverter and the ferroelectric transistor inverter.
\end{abstract}

Keywords: MOSFET, FeFET, ferroelectric transistor inverter 
Ferroelectric Inverter Circuit
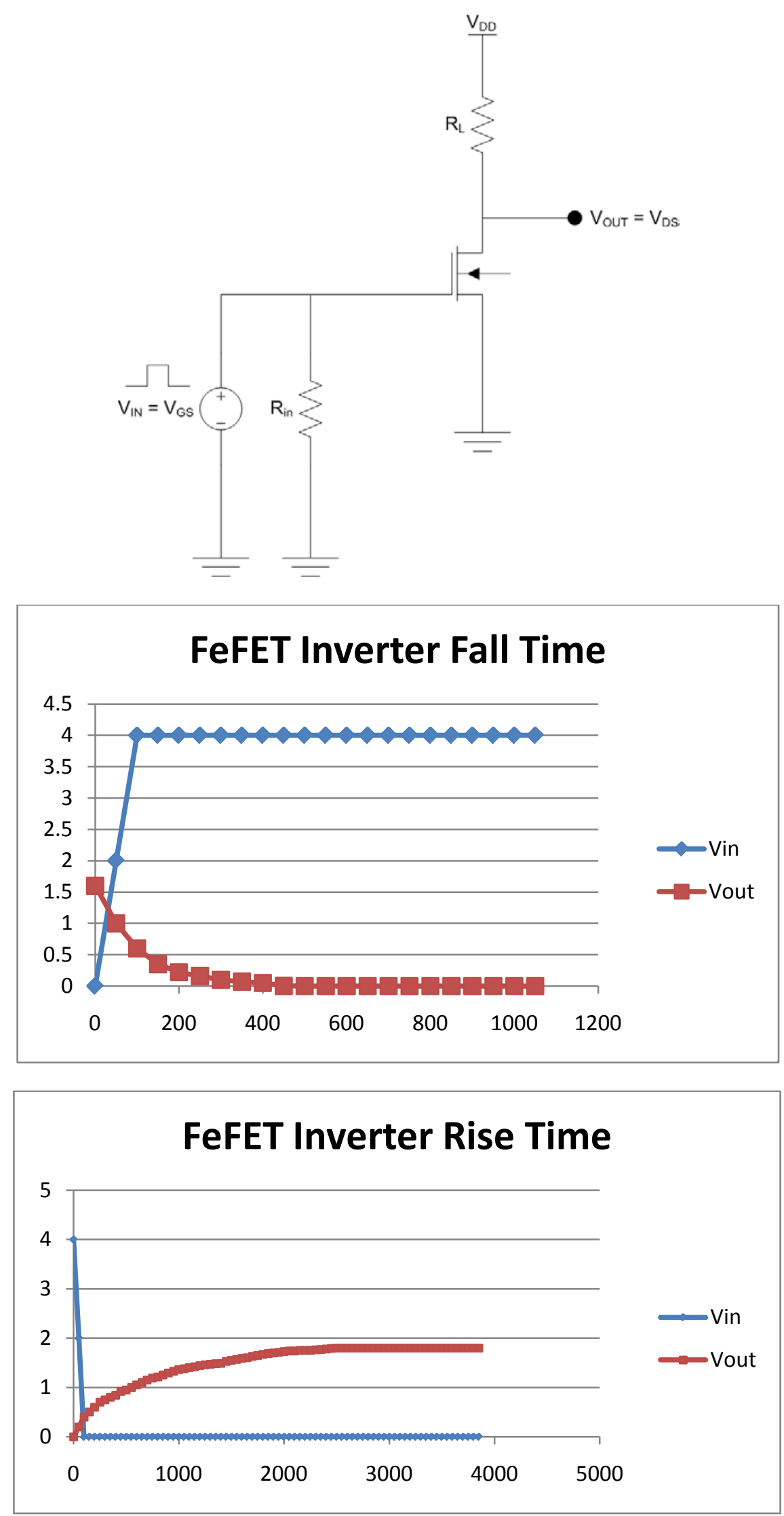